\title{
Hermite Matrix and Its Eigenvalue-based Decomposition
}

\author{
Baofa Sun ${ }^{*}$
}

Department of Computer Science and Technology, Anhui Sanlian University, Hefei, Anhui, China

\begin{abstract}
In the MUSIC approach for multiple emitter location, the array covariance matrix is a Hermite matrix. In order to realize the MUSIC approach, we have to do the work of eigenvalue-based decomposition of the Hermite matrix. This paper proves that the problem of Hermite matrix decomposition can be transformed into the problem of real symmetric matrix decomposition, and the article gives the detailed transformation method. Using Jacobi diagonalization method, the eigenvalue-based decomposition of real symmetric matrix decomposition is realized on computer, so the eigenvalue-based decomposition of a Hermite matrix is realized on computer.
\end{abstract}

Keywords: MUSIC approach, Hermite matrix, real symmetric matrix, eigenvalue-based decomposition of a matrix, Jacobi diagonalization method.

\section{INTRODUCTION}

At first, we introduce MUSIC approach for multiple emitter location briefly [1-3].

Let

$$
\begin{aligned}
& S(t)=\left(s_{1}(t), s_{2}(t), \cdots, s_{K}(t)\right)^{T}: \mathrm{K} \text { independent signals; } \\
& N(t)=\left(n_{1}(t), n_{2}(t), \cdots, n_{N}(t)\right)^{T}: \text { noise vector received by }
\end{aligned}
$$
uniform linear array with $\mathrm{N}$ antennas;

$$
X(t)=\left(x_{1}(t), x_{2}(t), \cdots, x_{N}(t)\right)^{T}: \text { signal vector received by }
$$
uniform linear array with $\mathrm{N}$ antennas; nals;

$A(\theta)=\left(a\left(\theta_{1}\right), a\left(\theta_{2}\right), \cdots, a\left(\theta_{K}\right)\right):$ direction matrix of sig-

$$
a\left(\theta_{i}\right)=\left(1, e^{j \frac{2 \pi d}{\lambda} \sin \theta_{i}}, \cdots, e^{j(N-1) \frac{2 \pi d}{\lambda} \sin \theta_{i}}\right)(i=1,2, \cdots, K): \text { di- }
$$

rection vector of the $i$ th signal,

then

$$
\begin{aligned}
& X(t)=A(\theta) S(t)+N(t), \\
& R_{x x}=E\left\{[X(t)-E(X(t))] \cdot[X(t)-E(X(t))]^{H}\right\} \\
& =E\left\{X(t) \cdot X(t)^{H}\right\} \\
& =A(\theta) E\left\{S(t) \cdot S^{H}(t)\right\} A^{H}(\theta)+E\left\{N(t) \cdot N^{H}(t)\right\} \\
& =A(\theta) P A^{H}(\theta)+\sigma^{2} I .
\end{aligned}
$$

Since the signal vector

*Address correspondence to this author at the Department of Computer Science and Technology, Anhui Sanlian University, Hefei, Anhui, China; Tel: 13856971057; E-mail: sunbaofa@sohu.com

$$
X(t)=\left(x_{1}(t), x_{2}(t), \cdots, x_{N}(t)\right)^{T}
$$

is complex, the array covariance matrix

$$
R_{x x}=R_{x x}(t)=E\left\{[X(t)-E(X(t))] \cdot[X(t)-E(X(t))]^{H}\right\}
$$

is a complex matrix.

Since $R_{x x}=R_{x x}(t)=A(\theta) P A^{H}(\theta)+\sigma^{2} I$, it is easy to prove that $R_{x x}^{H}=R_{x x}$, so $R_{x x}$ is a Hermite matrix.

For the complex matrix $R_{x x}$, the column of $R_{x x}$ can be viewed as $n$-dimensional vector in linear space $C^{n}$. The linear space generated by column vectors of $R_{x x}$ is called the matrix linear space of matrix $R_{x x}$ [4].

Decompose the matrix linear space $R_{x x}$ according to the eigenvalues, gain the linear space $E_{S}$ which contains the signal direction vectors $a\left(\theta_{i}\right)(i=1,2, \cdots, K)$ and the signal zero space $E_{N}$, where $R_{x x}=E_{S} \oplus E_{N}[4,5]$.

Define the spatial spectrum function

$$
P(\theta)=\frac{1}{\left\|a^{*}(\theta) \cdot E_{N}\right\|^{2}} .
$$

Scan the angle $\theta$ and observe the value of $P(\theta)$. Since $a\left(\theta_{i}\right) \perp E_{N}(i=1,2, \cdots, K), a^{*}\left(\theta_{i}\right) \cdot E_{N}=0, P(\theta)$ will have a peak when $\theta=\theta_{i}$. The DOA can be found according to the peak position $\theta_{i}$ of $P(\theta)$.

When we design the actual system for MUSIC approach, we always assume that the signals are short-time stable and the noise is stable, so $X(t)$ and $R_{x x}(t)$ are stable in short sampling time. In addition, the covariance function of general communication signals and Gauss white noise are er- 
godic, so the random process $R_{x x}(t)$ is ergodic. We can use the time-average of $R_{x x}(t)$ to replace the statistical-average of $R_{x x}(t)$, i.e. we can use

$$
\hat{R}_{x x}=\frac{1}{M} \sum_{m=1}^{M} R_{x x}\left(t_{m}\right)
$$

to replace $R_{x x}(t)$. According to the relation of $\hat{R}_{x x}$ and $R_{x x}(t), \hat{R}_{x x}$ is a Hermite matrix. In actual computation, we just do the eigenvalue-based decomposition of $\hat{R}_{x x}$.

In short, literatures [1-5] described the principle of MUSIC approach for multiple emitter location, and literatures [6-8] gave some specific simulation examples of MUSIC approach respectively. These literatures show that eigenvalue-based decomposition of the array covariance matrix is the basic task of MUSIC approach. Since the array covariance matrix is a Hermite matrix, so eigenvalue-based decomposition of Hermite matrix is very important for the MUSIC approach.

\section{THE WEAKNESS OF USUAL EIGENVALUE- BASED DECOMPOSITION METHOD}

The steps of usual eigenvalue-based decomposition of a matrix are as following [9-11]: at first, solve the eigenequation $\left|\lambda I-\hat{R}_{x x}\right|=0$ and get the eigenvalues of $\hat{R}_{x x}$; secondly, seek the eigenvectors belong to each eigenvalue.

In the array signal processing, the number of antennas, the order of array covariance matrix and the order of eigenequation are same, and the number of antennas is usually large. With the usual eigenvalue-based decomposition method, we have to seek the solutions of high order algebraic equation. However, according to the algebraic theory, the algebraic equation with the order more than 5 has no formula solutions. In general, it is very difficult to seek the eigenvalues of the array covariance matrix, sometimes we have to seek the approximate eigenvalues. Moreover, it is neither easy to seek the eigenvectors belonging to each eigenvalue, because we have to solve the high order linear equations $\left(\lambda I-\hat{R}_{x x}\right) q=0$. If $\lambda$ is the approximate eigenvalues, the linear equations have no solutions, then we must seek the least square solutions with more difficulty. It is visible that usual eigenvalue-based decomposition method is not suitable for MUSIC approach, we must look for other way of eigenvalue-based decomposition of Hermite matrix.

\section{THE RELATION OF TWO EIGENVALUE-BASED DECOMPOSITIONS}

There are many methods of real symmetric matrix decomposition. We hope to transform the problem of the Hermite matrix decomposition into the problem of real symmetric matrix decomposition.

Let $A$ be a $n$ order Hermite matrix, $A=A_{r}+i A_{i}$, real matrix $A_{r}$ and $A_{i}$ is the real part and the imaginary part of $A$ respectively. Since $A^{H}=A_{r}^{T}-i A_{i}^{T}$ and $A^{H}=A$,
$A_{r}^{T}=A_{r}, A_{i}^{T}=-A_{i}$, so $A_{r}$ is a real symmetric matrix and $A_{i}$ is a real antisymmetric matrix. Construct a $2 n$ order real matrix

$A^{\prime}=\left(\begin{array}{cc}A_{r} & -A_{i} \\ A_{i} & A_{r}\end{array}\right)$

For $A^{\prime T}=A^{\prime}, A^{\prime}$ is a real symmetric matrix. The relation of eigenvalue-based decomposition of Hermite matrix $A$ and real symmetric matrix $A^{\prime}$ is as following.

Theorem 1 Hermite matrix $A$ has the same eigenvalue set with the real symmetric matrix $A^{\prime}$.

Proof Let $\lambda$ be an eigenvalue of $A, q=q_{r}+i q_{i}$ be the eigenvector belonging to $\lambda$, then

$A q=\lambda q$,

$\left(A_{r}+i A_{i}\right)\left(q_{r}+i q_{i}\right)=\lambda\left(q_{r}+i q_{i}\right)$,

$\left(A_{r} q_{r}-A_{i} q_{i}\right)+i\left(A_{i} q_{r}+A_{r} q_{i}\right)=\lambda q_{r}+i \lambda q_{i}$,

$\left\{\begin{array}{l}A_{r} q_{r}-A_{i} q_{i}=\lambda q_{r}, \\ A_{i} q_{r}+A_{r} q_{i}=\lambda q_{i},\end{array}\right.$

$\left(\begin{array}{cc}A_{r} & -A_{i} \\ A_{i} & A_{r}\end{array}\right)\left(\begin{array}{l}q_{r} \\ q_{i}\end{array}\right)=\lambda\left(\begin{array}{l}q_{r} \\ q_{i}\end{array}\right)$

Let $q^{\prime}=\left(\begin{array}{l}q_{r} \\ q_{i}\end{array}\right)$, then $A^{\prime} q^{\prime}=\lambda q^{\prime}$. So $\lambda$ is an eigenvalue of $A^{\prime}$, and $q^{\prime}=\left(\begin{array}{l}q_{r} \\ q_{i}\end{array}\right)$ is the eigenvector of $A^{\prime}$ belonging to $\lambda$

Conversely, let $\lambda$ be an eigenvalue of $A^{\prime}, q^{\prime}=\left(\begin{array}{c}q_{r} \\ q_{i}\end{array}\right)$ be the eigenvector of $A^{\prime}$ belonging to $\lambda$, it is easy to prove that $\lambda$ is an eigenvalue of $A, q=q_{r}+i q_{i}$ is the eigenvector of $A$ belonging to $\lambda$.

In summary, Hermite matrix $A$ has the same eigenvalue set with the real symmetric matrix $A^{\prime}$.

Theorem 1 shows that eigenvalue-based decomposition of $n$ order Hermite matrix $A$ is equivalent to eigenvaluebased decomposition of $2 n$ order real symmetric matrix $A^{\prime}$. However, it is not difficult to find a problem: $n$ order Hermite matrix $A$ has $n$ eigenvalues, and $2 n$ order real symmetric matrix $A^{\prime}$ has $2 n$ eigenvalues, the eigenvalue numbers of two matrixes are not equal why do the two matrixes have the same eigenvalue set?

In order to have an intuitive understanding of the problem, we give an example to illustrate.

Hermite matrix

$A=\left(\begin{array}{cc}1 & -i \\ i & 1\end{array}\right)=\left(\begin{array}{ll}1 & 0 \\ 0 & 1\end{array}\right)+i\left(\begin{array}{cc}0 & -1 \\ 1 & 0\end{array}\right)$ 
has 2 eigenvalues, $\lambda_{1}=0, \lambda_{2}=2$.

The real symmetric matrix which is constructed by $A$

$$
A^{\prime}=\left(\begin{array}{cccc}
1 & 0 & 0 & 1 \\
0 & 1 & -1 & 0 \\
0 & -1 & 1 & 0 \\
1 & 0 & 0 & 1
\end{array}\right)
$$

has 4 eigenvalues, $\mu_{1}=\mu_{2}=0, \mu_{3}=\mu_{4}=2$.

The eigenvectors of $A^{\prime}$ belonging to eigenvalue $\mu_{1}=$ $\mu_{2}=0$ are

$q_{1}^{\prime}=\left(\begin{array}{c}1 \\ 0 \\ 0 \\ -1\end{array}\right), q_{2}^{\prime}=\left(\begin{array}{l}0 \\ 1 \\ 1 \\ 0\end{array}\right)$.

The eigenvectors of $A$ constructed by $q_{1}^{\prime}, q_{2}^{\prime}$ are

$q_{1}=\left(\begin{array}{l}1 \\ 0\end{array}\right)+i\left(\begin{array}{c}0 \\ -1\end{array}\right)=\left(\begin{array}{c}1 \\ -i\end{array}\right), q_{2}=\left(\begin{array}{l}0 \\ 1\end{array}\right)+i\left(\begin{array}{l}1 \\ 0\end{array}\right)=\left(\begin{array}{l}i \\ 1\end{array}\right)$.

Since $q_{2}=i q_{1}, q_{2}$ is the same eigenvector with $q_{1}$.

The example illustrates that $\lambda_{1}=0$, the 1 order eigenvalue of $\mathrm{A}$, corresponts to $\mu_{1}=\mu_{2}=0$, the 2 order eigenvalue of $A^{\prime}$, and $q_{1}$ (or $q_{2}$ ), the eigenvector of A belonging to eigenvalue $\lambda_{1}=0$, splits into 2 eigenvectors of $A^{\prime}$ belonging to eigenvalue $\mu_{1}=\mu_{2}=0, q_{1}^{\prime}$ and $q_{2}^{\prime}$.

Similarly, the eigenvalue $\lambda_{2}=2$ and the eigenvector of $A$ belonging to eigenvalue $\lambda_{2}=2$ has the same property.

Next, for general Hermite matrix $A, A^{\prime}$ is the real symmetric matrix constructed by $A$, we discuss the relation of the multiplicity of the eigenvalue $\lambda$ of $A$ and the multiplicity of the eigenvalue $\lambda$ of $A^{\prime}$.

Theorem 2 Let $\lambda$ be an eigenvalue of Hermite matrix $A$ and real symmetric matrix $A^{\prime}$, then the multiplicity of eigenvalue $\lambda$ in $A^{\prime}$ is double as in $A$.

Proof Let $q=q_{r}+i q_{i}$ be the eigenvector of $A$ belonging to $\lambda$, then $i q=-q_{i}+i q_{r}$ is the eigenvector of $A$ belonging to $\lambda$.

According to the proof of theorem 1,

$q_{1}^{\prime}=\left(\begin{array}{c}q_{r} \\ q_{i}\end{array}\right), \quad q_{2}^{\prime}=\left(\begin{array}{c}-q_{i} \\ q_{r}\end{array}\right)$

are all the eigenvectors of $A^{\prime}$ belonging to $\lambda$. Now we prove $q_{1}^{\prime}$ and $q_{2}^{\prime}$ are linear unrelative. Assume there are two real number $k_{1}$ and $k_{2}$, such that

$k_{1} q_{1}^{\prime}+k_{2} q_{2}^{\prime}=0$, or

$k_{1}\left(\begin{array}{c}q_{r} \\ q_{i}\end{array}\right)+k_{2}\left(\begin{array}{c}-q_{i} \\ q_{r}\end{array}\right)=0$,

i.e.

$\left\{\begin{array}{l}k_{1} q_{r}=k_{2} q_{i} \\ k_{1} q_{i}=-k_{2} q_{r} .\end{array}\right.$

Compute the inner product of $q_{r}$ and the first formula of Eq. (1), the inner product of $q_{i}$ and the second formula of Eq. (1), and get

$\left\{\begin{array}{l}k_{1}\left\|q_{r}\right\|^{2}=k_{2}\left(q_{i}, q_{r}\right), \\ k_{1}\left\|q_{i}\right\|^{2}=-k_{2}\left(q_{i}, q_{r}\right) .\end{array}\right.$

Add two eqations of Eq. (2), and get

$k_{1}\left(\left\|q_{r}\right\|^{2}+\left\|q_{i}\right\|^{2}\right)=0$.

Because $q_{1}^{\prime}=\left(\begin{array}{c}q_{r} \\ q_{i}\end{array}\right)$ is the eigenvector of $A^{\prime}$,

$\left\|q_{r}\right\|^{2}+\left\|q_{i}\right\|^{2} \neq 0$,

so $k_{1}=0$.

For the same reason, $k_{2}=0$.

Thus $q_{1}^{\prime}$ and $q_{2}^{\prime}$ are linear unrelative.

The argument shows that an eigenvector $q=q_{r}+i q_{i}$ of $A$ splits into two linear unrelative eigenvectors of $A^{\prime}$ :

$q_{1}^{\prime}=\left(\begin{array}{c}q_{r} \\ q_{i}\end{array}\right), q_{2}^{\prime}=\left(\begin{array}{c}-q_{i} \\ q_{r}\end{array}\right)$,

so the multiplicity of eigenvalue $\lambda$ in $A^{\prime}$ is double as in $A$.

According to theorem 1 and theorem 2, we can transform the problem of an $n$ order Hermite matrix decomposition into the problem of a $2 n$ order real symmetric matrix decomposition. Therefore, in order to do the work of eigenvalue-based decomposition of the Hermite matrix, we just need do the work of eigenvalue-based decomposition of real symmetric matrix. Next we discuss the eigenvalue-based decomposition of real symmetric matrix $[12,13]$.

\section{JACOBI DIAGONALIZATION METHOD}

There are many eigenvalue-based decomposition methods of real symmetric matrix, among them Jacobi diagonalization method is the most commonly used one.

In the program of Jacobi diagonalization transformation, each transformation of Jacobi diagonalization method eliminates a pair of off-diagonal elements of real symmetric matrix $A$. To eliminate a pair of off-diagonal elements $a_{p q}$ and $a_{q p}$, implement the orthogonal trans-formation to $A$ with orthogonal matrix 


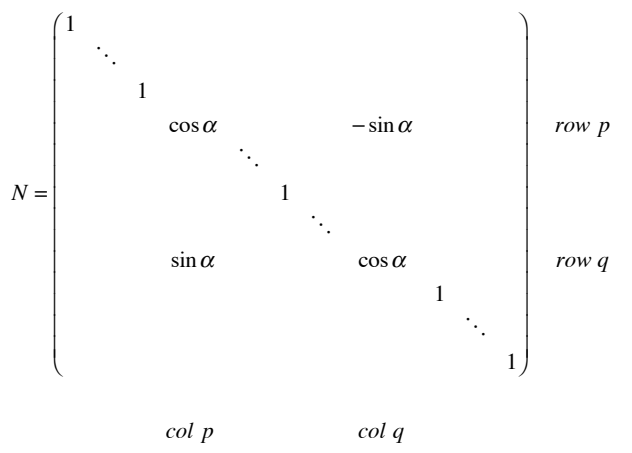

Select an $\alpha$ in the orthogonal transformation $N$ such that $\bar{a}_{p q}=\bar{a}_{q p}=0$ in $\bar{A}=N^{T} A N$. i.e. $\alpha$ meets the condition

$\bar{a}_{p q}=\left(-a_{p q}+a_{q q}\right) \cos \alpha \sin \alpha+a_{p q}\left(\cos ^{2} \alpha-\sin ^{2} \alpha\right)=0$

that is

$\tan 2 \alpha=\frac{2 a_{p q}}{a_{p p}-a_{q q}}$

In the orthogonal transformation $N$, it is not necessary to seek $\alpha$. However, we just need know $\sin \alpha$ and $\cos \alpha$, thus Eq.(3) can be rewritten as

$r^{2}=\left(a_{p p}-a_{q q}\right)^{2}+4 a_{p q}^{2}$

$\sin ^{2} \alpha=\frac{1}{2}-\frac{a_{p p}-a_{q q}}{2 r}$

$\cos ^{2} \alpha=\frac{1}{2}+\frac{a_{p p}-a_{q q}}{2 r}$

$\sin \alpha \cos \alpha=\frac{a_{p q}}{r}$

Calculate $r$ by Eq.(4a), calculate $\sin \alpha$ by Eq.(4b) and calculate $\cos \alpha$ by Eq.(4c) or Eq.(4d).

The orthogonal transformation $\bar{A}=N^{T} A N$ changes column $p$, column $q$, row $p$ and row $q$ of $A$ only, other columns and rows of $A$ are not changed. After the transformation, two diagonal elements are

$\bar{a}_{p p}=a_{p p} \cos ^{2} \alpha+a_{q q} \sin ^{2} \alpha+2 a_{p q} \sin \alpha \cos \alpha$

$=\frac{a_{p p}+a_{q q}+r}{2}$

$\bar{a}_{q q}=a_{p p} \sin ^{2} \alpha+a_{q q} \cos ^{2} \alpha-2 a_{p q} \sin \alpha \cos \alpha$

$=\frac{a_{p p}+a_{q q}-r}{2}$

Other elements of column $p$, column $q$, row $p$ and row $q$ are

$\bar{a}_{i p}=\bar{a}_{p i}=a_{i p} \cos \alpha+a_{i q} \sin \alpha$

$\bar{a}_{i q}=\bar{a}_{q i}=-a_{i p} \sin \alpha+a_{i q} \cos \alpha$ $(i=1,2, \cdots, n, \quad i \neq p, \quad i \neq q)$

In Eq. (5a) and Eq. (5b), $\bar{a}_{p p}>\bar{a}_{q q}$. For each orthogonal transformation, let $p<q$, then the eigenvalues in the last diagonal matrix will be in descending order.

A series of transform is needed to eliminate all offdiagonal elements generally. Because the eliminated elements do not always keep zeros, several iterations are required. Sign the $k$ transformation matrix as $N_{k}$, the gotten matrix of the $k$ transformation as $A^{(k)}$, then the $k+1$ transformation can be written as $A^{(k+1)}=N_{k+1}^{T} A^{(k)} N_{k+1}$. The eigenvectors matrix $Q^{(k+1)}$ of $A^{(k+1)}$ and the eigenvectors matrix $Q^{(k)}$ of $A^{(k)}$ satisfy $Q^{(k+1)}=N_{k+1}^{T} Q^{(k)}$.

Suppose there are $s$ transformations to diagonalize $A$, then $Q^{(s)}=N_{s}^{T} N_{s-1}^{T} \cdots N_{2}^{T} N_{1}^{T} Q$, where $Q$ is the eigenvectors matrix of $A$. For $Q^{(s)}$ is the eigenvectors matrix of diagonal matrix, $Q^{(s)}$ can be unit matrix $I$, i.e. $Q^{(s)}=I$. So

$Q=N_{1} N_{2} \cdots N_{s}$

\section{THE REALIZATION OF JACOBI DIAGONALIZA- TION METHOD ON COMPUTER}

There are three realization means of Jacobi diagonalization method on computer.

\section{(1) Tracking Search Method}

Each transformation makes the square-sum of the diagonal elements increase $2 a_{p q}^{2}$ while the square-sum of the offdiagonal elements decreases $2 a_{p q}^{2}$. If we select the largest module element of the off-diagonal elements for each transformation, then the efficiency to eliminate the off-diagonal elements is the highest.

Search for each row of the matrix, find out the largest module element of the off-diagonal elements, and record the column number of the element. The above information of the matrix is recorded in a $1 \times n$ real array and a $1 \times n$ integer array. So the largest module element of off-diagonal elements of the matrix can be found by searching for the real array, and the column number can be gotten from the corresponding position in the integer array. In the transformation process, these two arrays have to be revised continually to shorten the search process. If a transformation eliminate the element $a_{p q}$ and $a_{q p}$, the $p$ th element and the $q$ th element in two arrays must be modified.

It is easy to see that the search process will spend a lot of time to find out the largest module element of the offdiagonal elements after each transformation.

\section{(2) Jacobi Method in Order}

Each transformation doesn't eliminate the largest module element of off-diagonal elements, but eliminate the offdiagonal elements in certain order, such as $(p, q)=(1,2),(1$, $3), \ldots,(1, n),(2,3),(2,4), \ldots,(2, n), \ldots,(n-1, n)$. When all 
off-diagonal elements are eliminated once, repeat the process time and time again until each off-diagonal element is zero.

This method omits the searching process, it can overcome long-searching-time shortcomings of tracking search method, but it needs a lot of transformations and a large amount of calculation.

\section{(3) Jacobi Method with Threshold}

This method is the compromise between tracking search method and Jacobi method in order. By this method, a threshold is given, although the off-diagonal element is eliminated in order, only those off-diagonal elements whose module is larger than the threshold are eliminated, the offdiagonal elements whose module is lower than the threshold remain unchanged. When the modules of all off-diagonal elements are less than the threshold, reduce the threshold and repeat the process again. End the iterative process when the threshold is small enough.

We can actualize Jacobi method with threshold by the following steps.

At first, calculate the square root of square sum of the off-diagonal elements of $A$, and get

$v_{0}=\left(2 \sum_{i=1}^{n-1} \sum_{j=i+1}^{n} a_{i j}^{2}\right)^{\frac{1}{2}}$.

Secondly, set threshold $v_{1}=v_{0} / n$. Scan the off-diagonal elements of the matrix $A$ in accordance with the line and seek the first element $a_{p q}$ which module is larger than the threshold $v_{1}$, implement the orthogonal transfor-mation to $A$ with orthogonal matrix $\mathrm{N}$ to eliminate $a_{p q}$. Repeat the process again and again until the modules of all off-diagonal elements are less than the threshold $v_{1}$.

Thirdly, set threshold $v_{2}=v_{1} / n$ and repeat the process.

At last, end the iterative process when the threshold is small enough, e.g. $v_{k}<\varepsilon$.

We choose Jacobi method with threshold evaluating the effectiveness of above three methods comprehensively.

\section{THE STEPS OF EIGENVALUE-BASED DECOM- POSITION OF HERMITE MATRIX}

According to above discussion, we obtain the steps of eigenvalue-based decomposition of Hermite matrix $A$.

(1) Separate $A$ into real part and imaginary part

$A=A_{r}+i A_{i}$.

(2) Construct a real symmetric matrix

$$
A^{\prime}=\left(\begin{array}{cc}
A_{r} & -A_{i} \\
A_{i} & A_{r}
\end{array}\right)
$$

(3) Eliminate the off-diagonal elements of $A^{\prime}$ by Jacobi method with threshold, get a diagonal matrix. The diagonal elements of the matrix are all eigenvalues of $A^{\prime}$.

(4) All transformation matrixes $N_{i}$ sequentially multiply and get the eigenvector matrix of $A$ according to Eq. (7).

\section{CONCLUSIONS}

In the MUSIC approach, eigenvalue-based decomposition of the Hermite matrix is the basic work. By transforming the problem of Hermite matrix decomposition into the problem of real symmetric matrix decomposition, the basic work has been done.

Literatures $[7,8]$ gave some specific simulation examples of MUSIC approach, and the simulation results showed that the methods discussed in this article was correct, feasible and effective.

\section{CONFLICT OF INTEREST}

The authors confirm that this article content has no conflicts of interest.

\section{ACKNOWLEDGEMENTS}

Declared none.

\section{REFERENCES}

[1] R.O. Schmidt, "Multiple Emitter Location and Signal Parameter Estimation", IEEE. Trans. AP, vol. 34, no. 3, pp. 276-280, 1986.

[2] X.D. Zhang, "Modern Signal Processing"(Second Edition). Tsinghua University Press, 2002.

[3] D.S. Liu, J.Q. Luo, J.Y. Zhang, "Spatial spectrum estimation and its application", University of Science and Technology of China Press, China, 1997.

[4] B.F. Sun, "The mathematical standard of music approach for multiple emitter location", ITCAE 2013, 2013.

[5] B.F. Sun, "The eigenvalue-based decomposition method of Hermite matrix and its realization on computer", ICMII2013, 2013.

[6] R.O. Schmidt, R.E. Franks, "Multiple sources DF Signal processing: an experiment system", IEEE. Trans. AP, vol. 34, no. 3, 1986.

[7] B.F. Sun, "Visual simulation for determining unidirectional DOAs", $7^{\text {th }}$ ICEMI, pp. 692-695, 2005.

[8] B.F. Sun, "Simulation of spatial smoothing approach for coherent sources" DF, 1 1 ISTAI, pp. 1557-1561, 2006.

[9] Y.P. Cheng, "Matrix Theory", Northwestern Polytechnical University Press, China, 1999.

[10] J.H. Luo, "Introduction to matrix analysis", South China University of Technology Press, China 1992.

[11] Z.X. Jiang, G.L. Shi, "Matrix theory and its application”, Beijing Aviation College Press, China 1988.

[12] A. Jennings, "Matrix algorithms of engineering and science". Tsinghua University Press, China, 1985.

[13] C.F. Zhou, "Digital algorithms of science and engineering (Based on Visual C++)", Tsinghua University Press, China, 2002.

Received: August 13, 2013

Revised: August 28, 2013

Accepted: August 28, 2013

(c) Baofa Sun; Licensee Bentham Open.

This is an open access article licensed under the terms of the Creative Commons Attribution Non-Commercial License (http://creativecommons.org/licenses/by-nc/3.0/) which permits unrestricted, non-commercial use, distribution and reproduction in any medium, provided the work is properly cited. 